\section{Leukocytoclastic vasculitis associated with crusted scabies}

Leukocytoclastic vasculitis (LCV) is considered to be a hypersensitivity reaction mediated by immune complexes. The causes of LCV include infections, drugs, and systemic diseases [1]. An association between scabies and vasculitis has been reported [1-6]. Here, we report a patient who developed severe generalized cutaneous vasculitis after scabies infection.

A 74-year-old man presented with a two-week history of a progressive pruritic eruption on the trunk and limbs. Treatment with oral prednisone $(5 \mathrm{mg} /$ day) and a topical (Class II) steroid ointment had been prescribed by another skin clinic one week before, but generalized haemorrhagic skin lesions had then appeared and developed rapidly. His previous medical history included heart failure and arterial fibrillation caused by ischaemic heart disease. His medications included aspirin, warfarin, azosemide, diltiazem, and aldactone, but these had not been changed recently.

Physical examination revealed numerous purpuric macules or papules, $2-20 \mathrm{~mm}$ in diameter, over the entire body (figure $1 A, B$ ) and excoriated papules, crusts, and hyperkeratotic lesions on the buttocks (figure $1 C$ ). Many scabies mites and eggs were isolated from lesions of the buttocks. Histological examination of a purpuric lesion from the leg revealed LCV. The superficial dermis was infiltrated with inflammatory cells, including neutrophils, eosinophils, and lymphocytes (figure 1D); the cells were present both within and around the vessel walls (figure 1E). Red blood

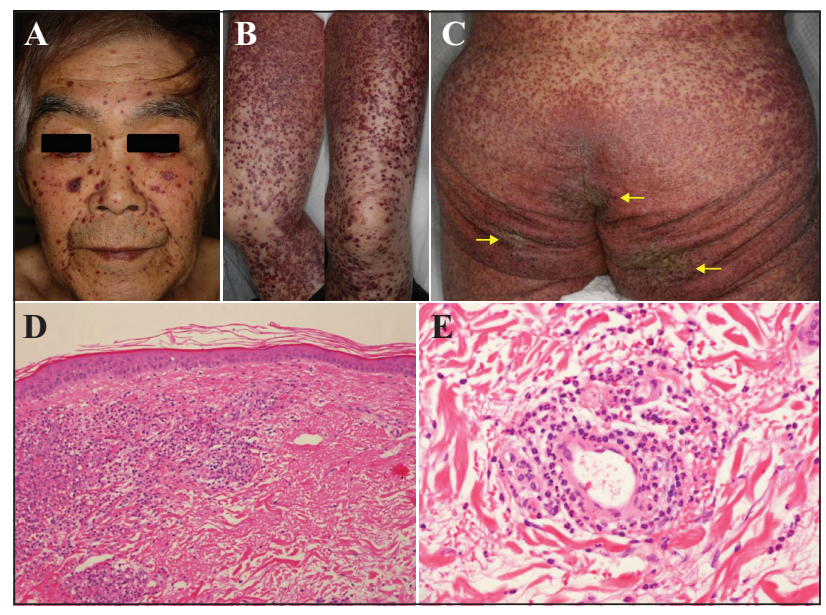

Figure 1. Numerous purpuric lesions over the entire body, including the face (A) and legs (B), and excoriated papules, crusts, and hyperkeratosis on the buttocks (C); numerous scabies mites and eggs were isolated from the lesions indicated by arrows. (D) Histological examination of a purpura sample from a leg reveals LCV; in the superficial dermis, many inflammatory cells infiltrate within and around vessels (haematoxylin-eosin stain; original magnification: $\times 40$ ). $(\mathbf{E})$ Inflammatory cells consist of neutrophils, eosinophils, and lymphocytes; extravasation of red blood cells, fibrinoid necrosis, and leukocytoclasia are also evident (haematoxylin-eosin stain; original magnification: $\times 100)$. cell extravasation was also apparent. Fibrinoid necrosis and leukocytoclasia were also evident (figure 1E). Blood tests revealed an elevated white blood cell count $\left(10,300\right.$ cells $\left./ \mathrm{mm}^{3}\right)$ and increased levels of C-reactive protein $(8.4 \mathrm{mg} / \mathrm{dL})$, fibrinogen $(430 \mathrm{mg} / \mathrm{dL})$, fibrin/fibrinogen degradation products $(19.8 \mu \mathrm{g} / \mathrm{mL}), \operatorname{IgA}(493 \mathrm{mg} / \mathrm{dL})$, and IgE $(682 \mathrm{IU} / \mathrm{mL})$. Platelet count was normal. The patient was negative for anti-ANCA and antinuclear antibodies. Urinalysis revealed intermittent microscopic haematuria and proteinuria. Treatment with four courses of oral ivermectin $(9 \mathrm{mg} /$ week) and topical phenothrin (one bottle/week) resolved the pruritus, and the papules and purpura resolved within two weeks. No additional papular or vasculitic skin lesions developed.

Scabies is a common skin infection caused by Sarcoptes scabiei var. hominis [3]. Clinically, scabies has several presentations: a classic acute infection, nodular scabies, and crusted scabies [7]. In our case, many excoriated papules, crusts, and hyperkeratosis were observed on the buttocks, from where many scabies mites and eggs were isolated. Therefore, we diagnosed our patient with crusted scabies. However, vascular purpura extending over the entire body is rare in the course of scabies.

The common histopathological findings of scabies include epidermal spongiosis, which can progress to vesiculation. The dermal infiltrate is usually composed of mixed perivascular inflammatory cells, including lymphocytes, histocytes, and numerous eosinophils. Occasionally, mites or eggs are seen in the stratum corneum. Vasculitis has rarely been reported [8, 9]. In our case, histological examination of the purpuric lesions revealed LCV. An association between scabies and LCV is uncommon, but LCV has been reported as a rare manifestation of scabies [1-6].

Nine cases of scabies with LCV have been reported [16] (table S1). No cause of vasculitis was identified for any patient, and none had a history of drug ingestion. In most cases, the purpura improved immediately after scabies treatment $[1-3,5]$, strongly supporting the contention that scabies caused the vasculitis.

In our patient, the differential diagnoses for LCV included IgA vasculitis and ANCA-associated, drug-induced septic vasculitis. Symptoms of IgA vasculitis, including arthralgia and gastralgia, were not reported, however, this cannot be excluded as no direct immunofluorescence assay was performed. The patient was negative for anti-ANCA antibodies and the diagnostic criteria for ANCA-associated vasculitis were not met. There was no history of drug ingestion which could have triggered drug-related vasculitis, however, the long-term treatment with an anticoagulant (warfarin) and an antiplatelet drug (aspirin) may have worsened the purpura. Septic vasculitis was not suspected because blood culture was negative. Repetitive intense scratching due to the severe itch may also have exacerbated the purpura.

In conclusion, we diagnosed this patient with LCV associated with crusted scabies. We speculate that scabies mites or some of their metabolic products (e.g. proteases) can trigger $\mathrm{LCV}$, possibly reflecting a hypersensitivity reaction against some immune complexes, the antigens of which remain to be identified.

Disclosure. Financial support: none. Conflict of interest: none. 


\section{Supplementary Data}

Supplementary Data (Table S1) along with the online version of this article is available on http://www. sciencedirect.com and doi: 10.1684/ejd.2017.3203.

Department of Dermatology, Ehime
University Graduate School of
Medicine, Toon, Ehime, Japan
<nishihara.katsuhiko.vr@ehime-
u.ac.jp>

Katsuhiko NISHIHARA Ken SHIRAISHI Koji SAYAMA

1. Valks R, Buezo GF, Dauden E. Scabies and leukocytoclastic vasculitis in an HIV-seropositive man. Int J Dermatol 1996;35: 605-6.

2. Clevy $C$, Brajon $D$, Combes $E$, et al. Scabietic vasculitis: report of 2 cases. Ann Dermatol Venereol 2017; 144: 349-55.

3. Stinco G, Governatori G, Interstimone D, Frattasio A, Patrone P. Scabetic leukocytoclastic vasculitis. Eur J Dermatol 2008; 18: 479-81. 4. Jarrett $P$, Snow J. Scabies presenting as a necrotizing vasculitis in the presence of lupus anticoagulant. Br J Dermatol 1998; 139:701-3. 5. Menne T, Christophersen J, Gram N, Bjerrehus T. Scabietic leucocytoclastic vasculitis with focal glomerulonephritis. Acta Derm Venereol 1984; 64: 445-7

6. Hay RJ. Norwegian scabies in a patient with a cutaneous vasculitis. Guys Hosp Rep 1974; 123: 177-85.

7. Elwood H, Berry RS, Gardner JM, Shalin SC. Superficial fibrin thrombi and other findings: a review of the histopathology of human scabietic infections. J Cutan Pathol 2015; 42:346-52.

8. Falk ES, Eide TJ. Histologic and clinical findings in human scabies. Int J Dermatol 1981; 20: 600-5.

9. Fernandez N, Torres $A$, Ackerman $A B$. Pathologic findings in human scabies. Arch Dermatol 1977; 113:320-4.

doi:10.1684/ejd.2017.3203

\section{DITRA syndrome in a Vietnamese patient: efficacy of etanercept}

More than 80 cases of generalized pustular psoriasis (GPP) associated with a mutation of the interleukin-36 receptor antagonist $(I L 36 R N)$ gene have been reported, and this syndrome is known as DITRA (deficiency of IL36RN). In patients with GPP and psoriasis vulgaris (PV), however, association with such mutation was not reported to be significant [1]. Fourteen different mutations of the IL36RN gene have been documented [2]; the c. 115+6C $>$ T mutation has only been described in Asian patients [3]. To date, no treatment has been approved for DITRA. We report a Vietnamese man with GPP associated with the c. $115+6 \mathrm{C}>\mathrm{T}$ mutation, who was effectively treated with the TNF-alpha inhibitor, etanercept, with complete remission for three years.

A 23-year-old man was hospitalised for a fifth episode of GPP (figure 1). The patient was Vietnamese, born to unrelated parents, and had no family history of psoriasis. Treatments with phototherapy, acitretin, methotrexate, and many topical corticosteroids had been inadequate. The flares were accompanied by generalized symptoms (fever at $39-40^{\circ} \mathrm{C}$, chills, and weakness) and biological signs of
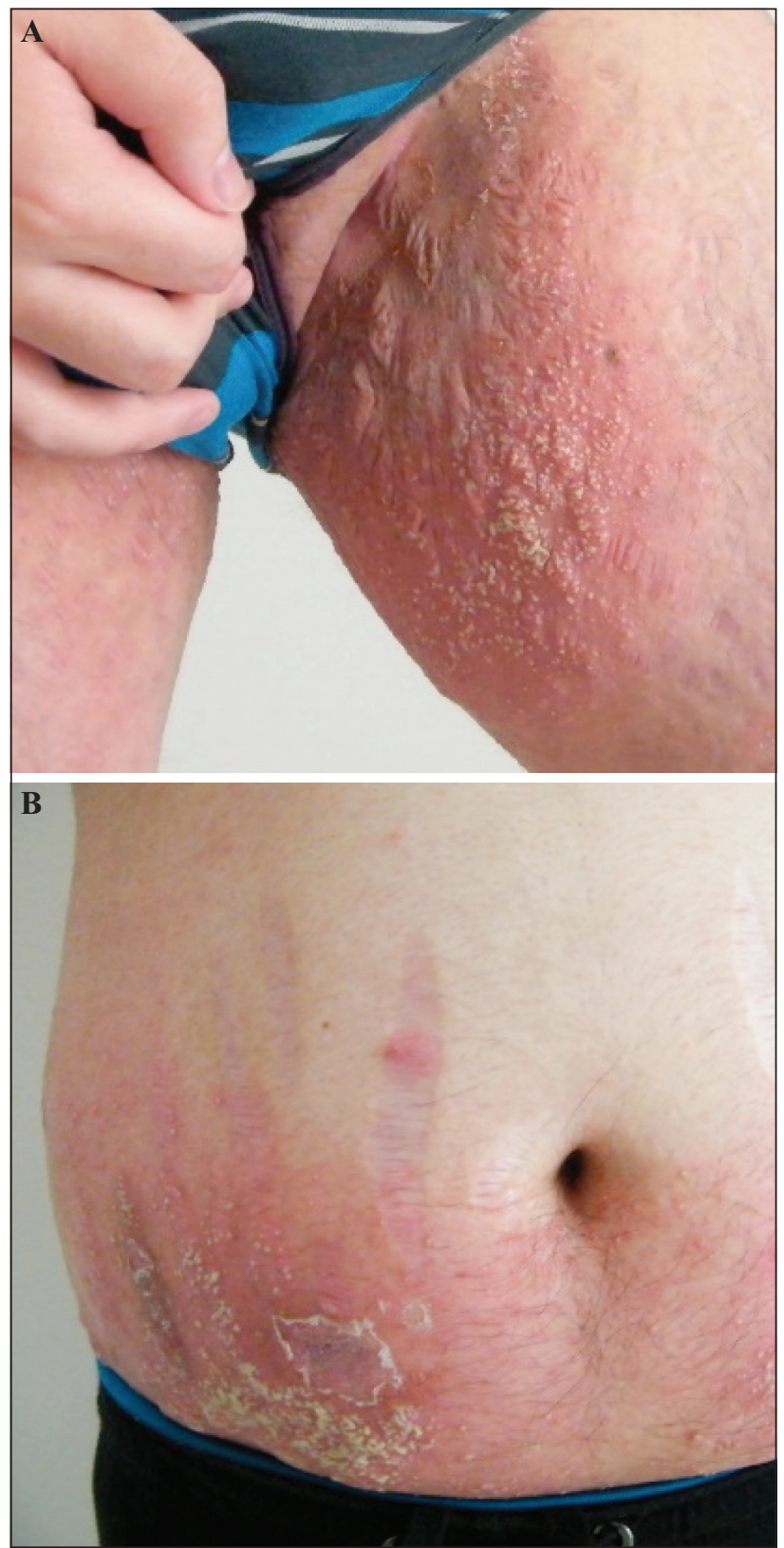

Figure 1. A) Pustular psoriasis of the left thigh. B) Abdominal pustular psoriasis.

inflammation. No infectious cause was found. The hypothesis of an auto-inflammatory disease was suggested. The c. $115+6 \mathrm{~T}>\mathrm{C}$ homozygous mutation of the IL36RN gene on chromosome 2q13 (indicating DITRA) was detected. TNF-alpha inhibitor treatment was initiated with $50 \mathrm{mg}$ etanercept administered twice weekly for 12 weeks, followed by $50 \mathrm{mg}$ per week. Significant improvement was rapidly noticed with disappearance of psoriasis within two weeks, leading to complete remission for a period of three years.

The pathogenesis of GPP is only partly understood. Pustular forms of psoriasis are rare. Following the recent discovery of mutations of the IL36RN gene, the pathogenesis of the disease is better understood. We believe that differences exist between the pathogenesis of GPP with and without 